Communications in Physics, Vol. 24, No. 3S2 (2014), pp. 133-138

DOI:10.15625/0868-3166/24/3S2/5059

\title{
EXPERIMENTAL DETERMINATION OF PHOTO LUMINESSCENSE QUANTUM YIELD OF CDSE QUANTUM DOTS
}

\author{
NGUYEN THANH BINH \\ Center for Quantum Electronics, Institute of Physics, Vietnam Academy of Science and \\ Technology \\ E-mail: tbnguyen@iop.vast.ac.vn
}

Received 20 June 2014

Accepted for publication 20 August 2014

\begin{abstract}
The fluorescence quantum yield or the efficiency of the fluorescence process is defined as the ratio of the number of photons emitted to the number of photons absorbed. In this report the experimental method to determinate photo luminescence $(P L)$ quantum yield $(Q Y)$ of CdSe quantum dot will be described. The experimental condition which depends on $Q Y$ will be investigated. The $Q Y$ values of $C d S e$ which were prepared at Nano Materials Physics Laboratory Research Center for Dielectric and Advanced Matters Physics Pusan National University will be carried out and compared with the commercial CdSe sample.
\end{abstract}

\section{INTRODUCTION}

Semiconductor quantum dots (QDs) have received tremendous attention due to considerable potential for a diverse range of technological applications. In contrast to many binary semiconductor quantum dots, CdSe QDs are readily synthesized with narrow size distributions and possess excellent photochemical stability when passivated properly [1,2]. In addition, they demonstrate size-dependent photoluminescence that encompasses the visible region of the electromagnetic spectrum, and high luminescence quantum yield. Hence, CdSe represents an archetypal system for numerous QD applications.

\section{Basics of fluorescence}

Fluorescence is a several-step process that begins when a relatively high-energy photon is absorbed by a system (typically an atom or molecule). The system may then release some of the energy internally (e.g. in increased vibrational motion of the atoms in a molecule). This is followed by a transition to an energy level close to the original one, with the emission of a photon; this photon constitutes the fluorescence emission. Since some of the absorbed photon's energy is released through internal conversion, the emitted (fluorescence) photon must have lower energy than the absorbed photon. This process is illustrated in the left-hand diagram in Fig. 1 below. This energy shift is known as Stokes' Law, and is commonly observed in fluorescent systems. In many 
commercial applications (e.g. enhanced "day-glo" colors), the absorbed photon is in the UV, while the emitted photon is in the visible region.
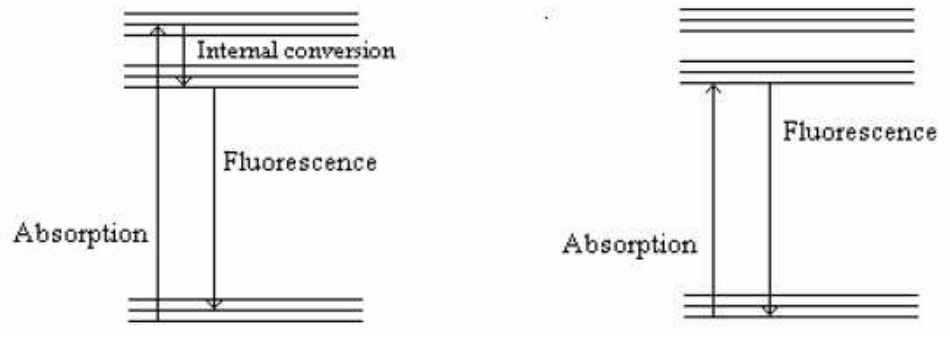

Fig. 1. Typical processes involving fluorescence

\section{Quantum yield}

The luminescence quantum yield is given as the ratio of the number of photons emitted to the number of photons absorbed by the sample:

$$
Q Y=\frac{\text { photon }_{\text {em. }} .}{\text { photon }_{\text {abs. }} .}
$$

Absolute measurements of QY are difficult, but it is straight forward to find the ratio of the QY for two substances, denoted by 1 and 2.

$$
Q Y 2 / Q Y 1=[F 2 / A 2] /[F 1 / A 1]=[F 2 / F 1][A 1 / A 2]
$$

where, $F 1$ is integrated intensity of the substance 1 fluorescence spectrum, $F 2$ is integrated intensity of the substance 2 fluorescence spectrum, $A 1$ is absorbance at wavelength $\lambda$ a for the substance 1 absorption spectrum, $A 2$ is absorbance at wavelength $\lambda$ a for the substance 2 absorption spectrum.

It is easier to determine the "relative" quantum yield of a fluorophore by comparing it to a standard with a known quantum yield. Some of the most common standards are listed in Table 1.

\section{EXPERIMENTAL}

The CdSe NQDs were synthesized with $\mathrm{CdO}$ and Se as precursors by the colloidal chemical process at Laboratory Physics of Nano Materials Pusan National University, Korea, an other commercial CdSe sample also was used to compare

Fluorescence spectra of CdSe QD arising from excitation by incident $\lambda_{a}$ light from xenon lamp which was dispersed by the first monochromator, the fluorescence signal was collected into second monochromator and detected by PMT. The reference sample is Rhodamine $6 \mathrm{G}$ with quantum yield about of $95 \%$. Absorption spectra will also be taken, including a quantitative determination of the absorbance at the UV excitation wavelength. The absorption and fluorescence spectra for the reference sample and for the quantum dots will be compared and their relationship examined in the light of the usual Stokes' law behavior. Also, the quantum yield of the quantum dot sample will be expressed in terms of the quantum yield of the dye standard and evaluated quantitatively based on the accepted value for the standard. 
Table 1. List of common standards for fluorescence quantum yield measurements.

\begin{tabular}{lclcc}
\hline $\begin{array}{l}\text { Quantum Yield [Q.Y.] } \\
\text { Standards }\end{array}$ & $\begin{array}{c}\text { Q.Y. } \\
{[\%]}\end{array}$ & $\begin{array}{l}\text { Conditions for } \\
\text { Q. Y. Measurements }\end{array}$ & $\begin{array}{c}\text { Excitation } \\
{[\mathrm{nm}]}\end{array}$ & Ref. \\
\hline Cy3 & 4 & PBS & 540 & {$[2]$} \\
Cy5 & 27 & PBS & 620 & {$[2]$} \\
Cresyl Violet & 53 & Methanol & 580 & {$[3]$} \\
Fluorescein & 95 & $0.1 \mathrm{M} \mathrm{NaOH}, 22^{\circ} \mathrm{C}$ & 496 & {$[3]$} \\
POPOP & 97 & Cyclohexane & 300 & {$[3]$} \\
Quinine sulfate & 58 & $0.1 \mathrm{M} \mathrm{H}_{2} \mathrm{SO}_{4}, 22^{\circ} \mathrm{C}$ & 350 & {$[3]$} \\
Rhodamine 101 & 100 & Ethanol & 450 & {$[4]$} \\
Rhodamine 6G & 95 & Water & 488 & {$[4]$} \\
Rhodamine B & 31 & Water & 514 & {$[4]$} \\
Tryptophan & 13 & Water, $20^{\circ} \mathrm{C}$ & 280 & {$[3,5]$} \\
L-Tyrosine & 14 & Water & 275 & {$[3,5]$} \\
\hline
\end{tabular}

The total number of fluorescence photons emitted is proportional to the area under the intensity vs wavelength curve; the software in the fluorometer performs that integration to yield the quantity F. The total number of incident photons that are absorbed in the fluorescence process is proportional to the absorbance $\mathrm{A}$ at wavelength $\lambda_{a}$, which is measured in a separate experiment using the UV-Vis absorption spectrometer. For best results in determining quantum yields, the two substances should have absorption peaks at approximately the same wavelengths, and their fluorescence spectra should overlap as much as possible.

\section{General Experimental Considerations}

Reference samples. The reference samples should be chosen to ensure they absorb at the excitation wavelength of choice for the test sample, and if possible, emit in a similar region to the test sample. The reference samples must be well characterised and suitable for such use.

Cuvettes. Standard $10 \mathrm{~mm}$ path length fluorescence cuvettes are sufficient for running the fluorescence measurements. In order to minimise errors in calculating the absorbance of each solution, it is advisable to use absorption cuvettes with extended path lengths

Concentration range. In order to minimise re-absorption effects absorbance in the $10 \mathrm{~mm}$ fluorescence cuvette should never exceed 0.05 at and above the excitation wavelength. Above this level, non-linear effects may be observed due to inner filter effects, and the resulting quantum yield values may be perturbed.

\section{RUSULT AND DISCUSSION}

\section{Calculation}

The relative quantum yield is generally determined by comparing the wavelength-integrated intensity of an unknown sample to that of a standard. The quantum yield of the unknown sample is calculated using:

$$
Q=Q_{R} \frac{I}{I_{R}} \cdot \frac{O D_{R}}{O D} \cdot \frac{n^{2}}{n_{R}^{2}}
$$


where $Q$ is the quantum yield, $I$ is the integrated intensity, $n$ is the refractive index, and $O D$ is the optical density. The subscript $R$ refers to the reference fluorophore of known quantum yield.

Fig. 2 show the absorption spectra and fluorescence spectra of CdSe and R6G. These spectra were recorded in the same condition. The line 488 indicate the excited wavelength for fluorescence spectra. The QY depend on excited wavelength and slit width of monochormator were investigated.
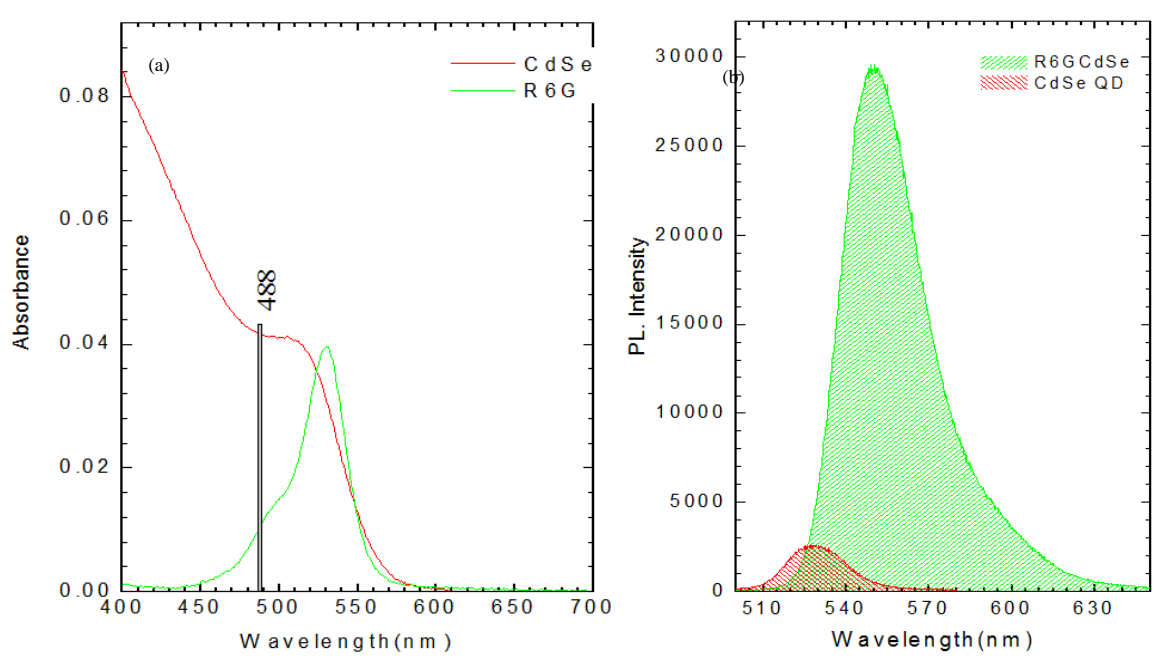

Fig. 2. Absorption spectra of CdSe and R6G (a) and Fluorescence spectra of CdSe and R6G (b), line 488 indicate the excited wavelength

\section{QY depend on excited wavelength}

Fluorescence spectra of CdSe depend on excited wavelength, QY of CdSe QD were calculated with different excited wavelength. Figure 3 show fluorescence spectra of CdSe excited by different wavelength from 420 to $540 \mathrm{~nm}$. The shape of spectra were changed but QY of CdSe (Fig. 4) almost do not change in region $470-520 \mathrm{~nm}$.

Table 2. Comparison QY of CdSe QD prepared at laboratory and CdSe QD commercial

\begin{tabular}{|c|c|c|}
\hline No & Sample & QY \\
\hline 1 & CdSe ODE 12 & 0.071 \\
\hline 2 & CdSe ODE 14 & 0.022 \\
\hline 3 & CdSe ODE 25 & 0.139 \\
\hline 4 & CdSe ODE x5 & 0.077 \\
\hline 5 & CdSe ODE 5-1 & 0.08 \\
\hline 6 & CdSe ODE 16 & 0.034 \\
\hline 7 & CdSe ODE ex. & 0.026 \\
\hline 8 & CdSe (Comm.) & 0.913 \\
\hline
\end{tabular}



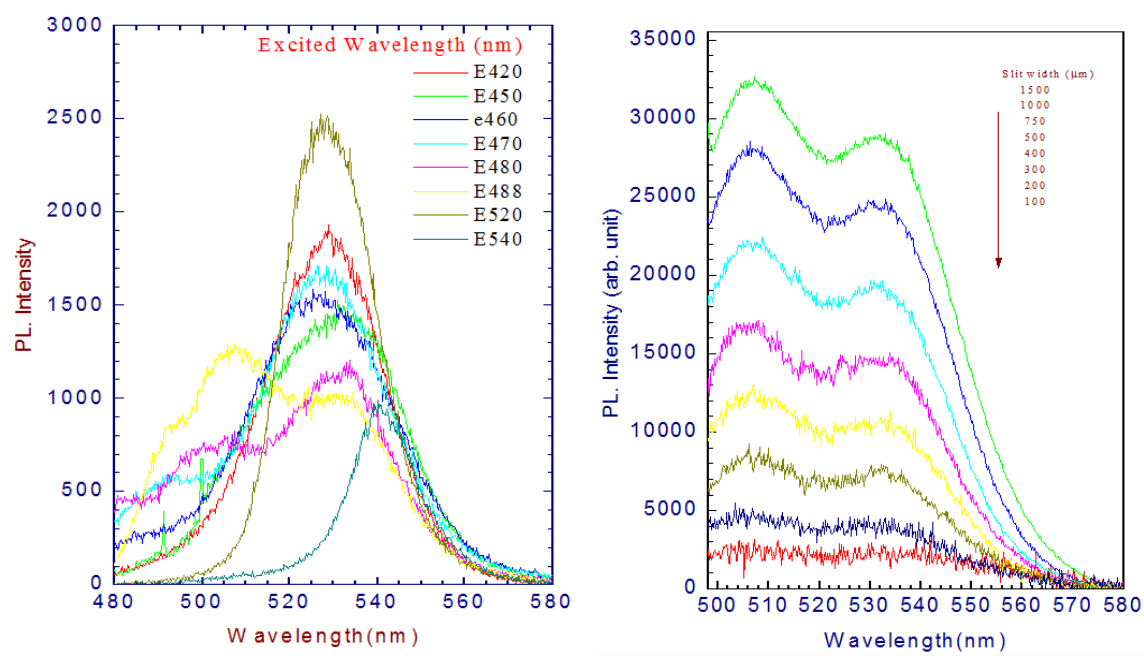

Fig. 3. Fluorescence spectra of CdSe QD measured with different excited wavelength (a) and different slit widths of monochromator (b)

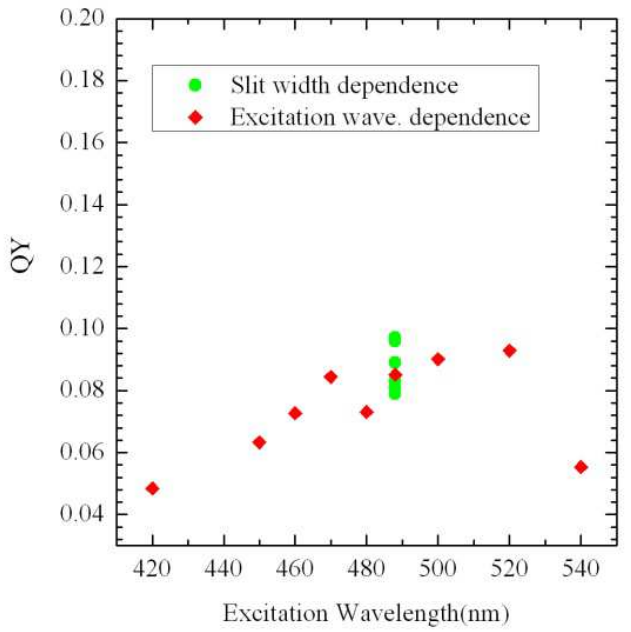

Fig. 4. QY of CdSe depend on excited wavelength and slit width

\section{CONCLUSION}

Measurement of fluorescence QY can often be difficult and troublesome, and the need for absolute care during every step cannot be over emphasised. However, it is possible to make such measurements routinely, and we were success in investigation, determination QY of CdSe QD. 


\section{ACKNOLEGMENTS}

This work was supported be National program on Fundamental research oriented applications, Contact No: G/07/2012/H-HUD and Intenational collaboration between Institute of Physics (VAST) and Pusan University (Korea).

\section{REFERENCES}

[1] C. B. Murray, D. J. Norris, and M. G. Bawendi, J. Am. Chem. Soc. 115 (1993) 8706.

[2] M. A. Hines and P. Guyot-Sionnest, J. Phys. Chem. 100 (1996) 468.

[3] R. B. Mujumdar, L. A. Ernst, S. R. Mujumdar, C. J. Lewis, A. S. Waggoner, Bioconj. Chem. 4 (1993) 105-111,

[4] J. R. Lakowicz, Principles of Fluorescence Spectroscopy, 2nd Ed., Kluwer Academic/Plenum Publishers, New York, London, Moscow, Dordrecht, 1999.

[5] D. Magde, G. E. Rojas, and P. Seybold, Photochem. Photobiol. 70 (1999) 737. 